\title{
Multimodal Prehabilitation and Major Complications after Colorectal Surgery for Cancer: The impact of Improving Preoperative Functional Capacity.
}

\author{
Minnella $\mathrm{EM}^{1}{ }^{1,2}$, Awasthi $\mathrm{R}^{1}$, Busquet-Dion $\mathrm{G}^{1}$, Loiselle S-E${ }^{1}$, Carli $\mathrm{F}^{1}$ \\ ${ }^{1}$ McGill University, Dept. of Anesthesia, Montreal, Quebec, Canada \\ 2University of Milan, Dept. of Anesthesia \& Intensive Care, Milan, Italy
}

\section{Background}

Multidisciplinary consensus agrees on the necessity to reduce major complications after abdominal surgery, as these represent a significant burden to patients and society. Multimodal prehabilitation programs comprising physical exercise, nutritional and anxiety-coping interventions have been shown to improve perioperative functional capacity in a greater proportion of patients. The impact of this improvement on major complications has not been investigated yet. The purpose of this study is to determine whether a preoperative functional improvement reduces the incidence of major complications after colorectal surgery.

\section{Methods}

This study involved a sample of 109 participants enrolled in one pilot and two randomized controlled trials. All participant underwent a 4-week multimodal prehabilitation program before colorectal surgery. Functional capacity was assessed with a six-minute walk test, a validated measure of surgical recovery that measure the distance walked in 6 minutes (6MWD).

As the minimal clinically important difference of 6MWD is estimated at $20 \mathrm{~m}$, two groups were defined as follows: one included participants whose change in 6MWD from baseline was less than $20 \mathrm{~m}(\mathrm{n}=48)$ and the other included those whose change in 6MWD was at least 20 m (n=61). Complication severity was graded according to the Clavien-Dindo classification, and those graded as III-V were defined as major. Primary outcome was the incidence of major complications.

\begin{tabular}{|ccc|}
\hline $\begin{array}{c}\text { Change in 6MWD } \\
\geq 20 ~ \mathbf{~}\end{array}$ & $\begin{array}{c}\text { Clavien-Dindo Grade } \\
\geq 3, \mathrm{n}(\%)\end{array}$ & P-value \\
\hline NO (n= 48) & $6(13)$ & \\
YES $(\mathrm{n}=\mathbf{6 1})$ & $1(2)$ & 0.042 \\
\hline
\end{tabular}

Results

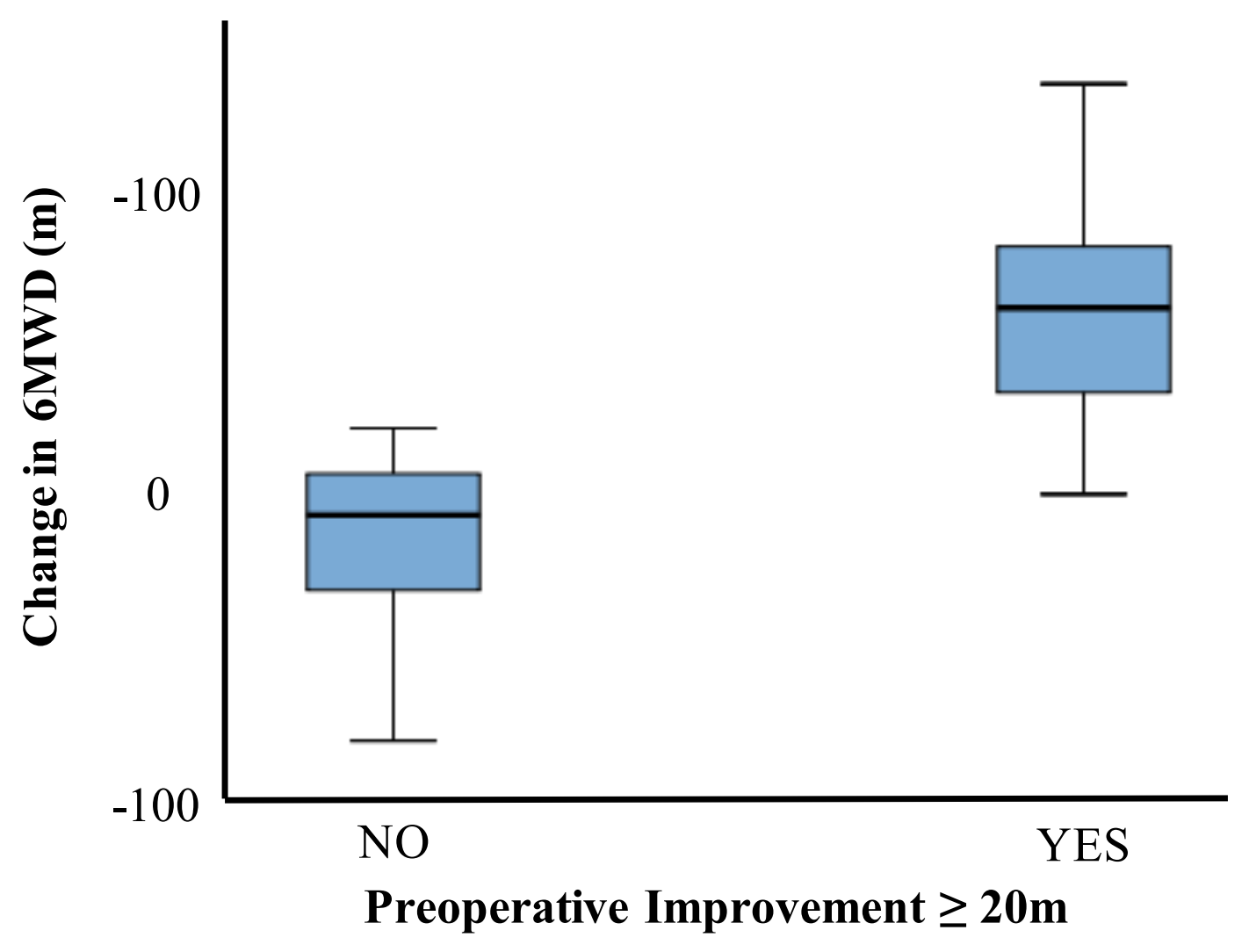

Preoperative Improvement $\geq 20 \mathrm{~m}$

There were no significant baseline differences between the groups for age, gender, BMI, ASA, Charlson Comorbidity Index, CR-POSSUM scores, cancer stage, surgical procedure and approach, duration of surgery and intraoperative blood loss. Participants experiencing a significant preoperative functional improvement had a lower baseline 6MWD (406.8 [IQR 377.6- 436.0] m vs. 448.0 [IQR 419.2-477.0] m, $\mathrm{p}=0.05$ ). There were $6(13 \%)$ major complications among subjects with a change in 6MWD below $20 \mathrm{~m}$, and one (2\%) in the other group ( $\mathrm{p}=0.042)$.

\section{Conclusion}

Preliminary data indicate that patients who achieved a preoperative significantly improvement in functional walking capacity are more likely to have less major complications after colorectal cancer surgery. 